\title{
GEOMETRIC INTEGRATORS FOR CLASSICAL SPIN SYSTEMS
}

\author{
JASON FRANK $\dagger$, WEIZHANG HUANG $\ddagger$, AND BENEDICT LEIMKUHLER ${ }^{\S}$
}

\begin{abstract}
Practical, structure-preserving methods for integrating classical Heisenberg spin systems are discussed. Two new integrators are derived and compared, including (1) a symmetric energy and spin-length preserving integrator based on a Red-Black splitting of the spin sites combined with a staggered timestepping scheme and (2) a (Lie-Poisson) symplectic integrator based on Hamiltonian splitting. The methods are applied to both $1 \mathrm{D}$ and $2 \mathrm{D}$ lattice models and are compared with the commonly used explicit Runge-Kutta, projected Runge-Kutta, and implicit midpoint schemes on the bases of accuracy, conservation of invariants and computational expense. It is shown that while any of the symmetry-preserving schemes improves the integration of the dynamics of solitons or vortex pairs compared to Runge-Kutta or projected Runge Kutta methods, the staggered Red-Black scheme is far more efficient than the other alternatives.
\end{abstract}

Key words. Lattice Landau-Lifshitz equation, Heisenberg spins, symplectic integrators, vortex dynamics.

1. Introduction. Recent studies of algorithms for conservative nonlinear systems arising in simulations of celestial mechanics [1], rigid body motion [2], and partial differential equations (PDEs) [11, 17] have proposed methods that preserve geometric features, such as time-reversal symmetry or symplectic structure, present in the equations of motion. Improved energy conservation in long term simulations and improved resolution of global or very long term solution behavior are some of the hoped for benefits from the use of geometric integrators. Despite the obvious aesthetic appeal of using a geometric integrator as opposed to an integrator that preserves none of the available structure, the question of the practical importance of geometric integrators in numerical simulations has so far remained largely open. For the very large systems arising from the discretization of partial differential equations in two or three dimensions, efficiency issues become extremely important, and the standard geometric integration schemes-which are typically implicit—can be prohibitively expensive to apply to complex nonlinear models. This article addresses issues of practicality and efficiency in the numerical solution of a conservative nonlinear partial differential equation, the Landau-Lifshitz (LL) equation. This system possesses integral invariants, a symplectic structure and a time symmetry; we compare implicit and semi-explicit methods that preserve each of these layers of structure.

The LL equation, originally proposed as a model of a continuous anisotropic Heisenberg ferromagnet, is given by

$$
\frac{\partial S}{\partial t}=S \times \nabla^{2} S+S \times D S
$$

where $S=S(x, y, t) \in \mathbf{R}^{3},\|S\|_{2}=1$. The matrix $D \in \mathbf{R}^{3 \times 3}$ represents the anisotropy and may be assumed to be diagonal, $D=\operatorname{diag}\left(d_{1}, d_{2}, d_{3}\right)$, and $\left|d_{1}\right| \leq\left|d_{2}\right| \leq\left|d_{3}\right|$. This equation has been described in [6] as a "universal integrable system" since various known integrable PDEs such as the nonlinear Schrödinger equation and sine-Gordon equation can be derived from its one space dimensional version as limiting cases. Variants of the LL equation-and discrete analogues-are important to understanding the properties of magnetic materials and have been the subject of recent numerical studies appearing in the physical literature $[14,22,23]$.

In $\S 2$, we describe integrals and symmetries of the lattice version of the Landau-Lifshitz equation obtained as spatial finite difference approximations of the LL equation. Integral conservation will be one basis for our comparison of various numerical methods. The presence of integral invariants such as the spin lengths

\footnotetext{
$\dagger$ Department of Technical Mathematics and Computer Science, Delft University of Technology, Delft, the Netherlands. (frank@math.tudelft.nl)

$\ddagger$ This author was supported in part by the National Science Foundation under EPSCoR grant OSR-9255223. Department of Mathematics, the University of Kansas, Lawrence, KS 66045, U.S.A. (huang@math.ukans.edu)

$\S$ Support for this research was provided by the National Science Foundation, grant number NSF-9303223. Department of Mathematics, the University of Kansas, Lawrence, KS 66045, U.S.A. (leimkuhl@math.ukans.edu)
} 
raises the question of whether integration would not better be performed on the reduced system obtained by parameterizing the constraints. Although such an approach is possible in canonical coordinate charts, it is not very practical for reasons outlined in $[9,10]$. In particular, substantial additional complexity is introduced in the computation of the vector field, an operation that must be carried out one or more times at each timestep. Also in $\S 2$, standard timestepping schemes for the semi-discrete system are described. Two geometric integrators are developed in $\S 3$ by splitting either the Hamiltonian or the vector field to decouple the system into small integrable systems. The conservative properties of these methods are also discussed in the section. In $\S 4$ we compare the various schemes, first for the integration of a known periodic solution of the $1 \mathrm{D}$ system, and second for the time evolution of $2 \mathrm{D}$ anisotropic vortex systems. Finally $\S 5$ is devoted to conclusions.

2. Lattice Landau-Lifshitz and related systems. In this section we consider a spatial discretization of equation (1). For simplicity, we assume that equation (1) is defined either on a one-dimensional domain $\Omega=$ $(-L, L)$ or on a rectangular domain $\Omega=(-L, L) \times(-L, L)$ in two dimensions, supplemented by periodic or homogeneous Dirichlet boundary conditions. We also work on a uniform mesh, $\left\{x_{i}=-L+i h, i=0, \ldots, N\right\}$ in one dimension or $\left\{\left(x_{i}, y_{j}\right) \equiv(-L+i h,-L+j h), i, j=0, \cdots, N\right\}$ in two dimensions, where $h=\frac{2 L}{N}$ and $N$ is a certain positive even integer.

The simplest discrete form of the LL equation is the one-dimensional isotropic Heisenberg spin chain:

$$
\frac{d}{d t} S_{i}=\frac{1}{h^{2}} S_{i} \times\left(S_{i-1}+S_{i+1}\right) .
$$

With periodic boundary conditions, this system has an exact solution [16, 20]

$$
S_{i}(t)=\left(a \cos \theta_{i}+b \sin \theta_{i}\right) \cos \phi+c \sin \phi,
$$

where

$$
\theta_{i}=i p-\omega t, \omega=2(1-\cos p) \sin \phi
$$

and $a, b, c \in \mathbf{R}^{3}$ form a right-handed set of orthogonal unit vectors, $\phi, p \in \mathbf{R}$ are scalar constants.

The $2 \mathrm{D}$ equation (1) can be discretized using second order central differences,

$$
\frac{d}{d t} S_{i j}=S_{i j} \times \frac{1}{h^{2}}\left(S_{i, j-1}+S_{i, j+1}+S_{i-1, j}+S_{i+1, j}\right)+S_{i j} \times D S_{i j}
$$

where $S_{i j}(t) \approx S(i h, j h, t)$. The boundary conditions are incorporated by either (periodic case):

$$
S_{i, N+1} \equiv S_{i, 1}, \quad S_{i, 0} \equiv S_{i, N}, \quad S_{N+1, j} \equiv S_{1, j}, \quad S_{0, j} \equiv S_{N, j}
$$

for $0 \leq i, j \leq N$, or (homogeneous Dirichlet case)

$$
S_{i, N+1} \equiv 0, \quad S_{i, 0} \equiv 0, \quad S_{N+1, j} \equiv 0, \quad S_{0, j} \equiv 0 .
$$

Equation (4) with $h=1$ will be referred to as the lattice Landau-Lifshitz equation.

For the numerical experiments of this paper we have used an alternative form of the semi-discrete system for equation (1). Replacing $S_{i j}$ in the term $D S_{i j}$ in equation (4) by an average, viz.,

$$
S_{i j}=\frac{1}{4}\left(S_{i, j-1}+S_{i, j+1}+S_{i-1, j}+S_{i+1, j}\right),
$$

results in

$$
\frac{d}{d t} S_{i, j}=S_{i, j} \times M\left(S_{i, j-1}+S_{i, j+1}+S_{i-1, j}+S_{i+1, j}\right),
$$


where $M \equiv I / h^{2}+D / 4$ is again a diagonal matrix. It is easy to see that (7) is also a second order approximation to equation (1). After [16], we will refer to this as the Roberts discretization.

The system (4) or (7) can always be rewritten in the form of the lattice Landau-Lifshitz equation (i.e. the system with $h=1$ ) by rescaling $S_{i, j}$ and the the anisotropic matrix D without altering the dynamical properties. Thus, we will always assume $h=1$ in the following discussion. We refer to the discrete $S_{i j}$ as spin vectors. Equation (7) is fairly general and allows for simulations of many physical phenomena [14, 24]. For example, the easy-plane anisotropy spin system considered in [24] can be described by equation (7) with

$$
M=\left[\begin{array}{ccc}
1 & 0 & 0 \\
0 & 1 & 0 \\
0 & 0 & 1-\delta
\end{array}\right]
$$

where $\delta$ is a parameter in $(0,1)$. This system admits stable vortex solutions.

2.1. Properties of the equations. We next enumerate some of the important features of the spin system (7) that make it an excellent test case for studying the relevance of geometric aspects for the design of numerical integrators. These properties include a Hamiltonian structure, various conserved quantities, and a scaling symmetry.

We will use the following notation: for a vector $u \in \mathbf{R}^{3}$, associate a $3 \times 3$ skew-symmetric matrix skew $(u)$ such that, for any other $v \in \mathbf{R}^{3}, v \times u \equiv \operatorname{skew}(u) v$.

Define a vector $S$ based on the natural ordering of the grid points:

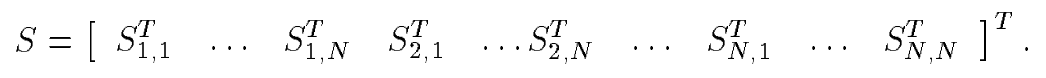

It is easy to verify that equation (7) can be written as a (Lie-Poisson) Hamiltonian evolution equation [13]

$$
\frac{d}{d t} S=J \nabla_{S} H,
$$

for the dependent variable $S$, with Hamiltonian

$$
H=-\sum_{i, j} S_{i, j} \cdot M\left(S_{i, j-1}+S_{i, j+1}+S_{i-1, j}+S_{i+1, j}\right),
$$

and the block diagonal symplectic structure matrix $J=J(S)$ consisting of blocks skew $\left(S_{i, j}\right)$ enumerated sequentially along the diagonal consistent with the natural ordering of the unknowns.

The Poisson bracket is defined (for any pair of smooth functions $f$ and $g$ of $S$ ) by

$$
\{f, g\}:=\nabla f^{T} J \nabla g .
$$

This bracket obeys the Jacobi identity:

$$
\{\{f, g\}, h\}+\{\{g, h\}, f\}+\{\{h, f\}, g\}=0
$$

for $C^{2}$ functions $f, g$, and $h$.

The fact that (7) is Hamiltonian implies that the flow map $\Phi_{t}$ (which evolves points of phase space through $t$ units of time) is symplectic; in other words,

$$
\Phi_{t}^{\prime T} J \Phi_{t}^{\prime}=J
$$

This is a strong invariant property of the flow map and one which has recently been studied in the context of numerical integration schemes [8, 18].

The systems (7), though not fully integrable, do possess certain obvious integral invariants: 
(i) The individual spin lengths are conserved, $\left\|S_{i, j}\right\|_{2}=$ constant.

(ii) The total spin, $\sum_{i} S_{i}$, provides three additional invariants in the isotropic case of periodic boundary conditions.

(iii) The Hamiltonian is of course always an integral.

In certain circumstances, there may be additional integrals of the motion, such as the discrete gyrovector mentioned in [24].

Next we consider scale invariance of the discrete systems. Suppose $S=S(t)$ is a solution of the system (7), and consider a scaling of time given by $t^{*}=t / \alpha$. We try to find a $\beta$ such that $S^{*}\left(t^{*}\right) \equiv \beta S(t)=\beta S\left(\alpha t^{*}\right)$ is also a solution of (7) (in the new coordinate $t^{*}$ ). For such a $\beta$ we must have,

$$
\begin{aligned}
\frac{d}{d t^{*}} S_{i j}^{*}\left(t^{*}\right) & =\beta\left(\frac{d}{d t} S_{i j}\right) \frac{d t}{d t^{*}}=\alpha \beta \frac{d}{d t} S_{i j}(t) \\
& =\alpha \beta S_{i j}(t) \times M\left(S_{i, j-1}(t)+S_{i, j+1}(t)+S_{i-1, j}(t)+S_{i+1, j}(t)\right) \\
& =\frac{\alpha}{\beta} S_{i j}^{*}\left(t^{*}\right) \times M\left(S_{i, j-1}^{*}(t)+S_{i, j+1}^{*}(t)+S_{i-1, j}^{*}(t)+S_{i+1, j}^{*}(t)\right)
\end{aligned}
$$

Thus, $S^{*}\left(t^{*}\right)$ is a solution when $\beta=\alpha$, and we have just shown

Theorem 1 (Scale invariance). If $S(t)$ is a solution of $(7)$, so is $\alpha S(\alpha t)$.

As before, let $\Phi_{t}$ be the flow map. Taking $\alpha=-1$, the scaling relation implies, by uniqueness, that $\Phi_{t}(S)=-\Phi_{-t}(-S)$ for an arbitrary vector $S$, or, since $\Phi_{-t}$ is the inverse of the flow map, that

$$
\Phi_{t}(S)=-\Phi_{t}^{-1}(-S), \quad \forall S \in \mathbf{R}^{3} .
$$

We do not know of any time integration methods that preserve the general form of the scaling law, but the time-symmetry (13) is maintained by a number of different methods.

2.2. Commonly used timestepping schemes. We restrict our attention to one-step numerical integration schemes, i.e. schemes that can be viewed as approximations $\hat{\Phi}_{t}$ of the flow map.

The commonly used methods for integrating the Landau-Lifshitz equation and its variants are explicit Runge-Kutta methods, which are relatively fast but have poor conservation properties. Recent studies of various nonlinear systems (see, e.g., [5]) have suggested using the implicit midpoint rule, which has much better conservation properties but is computationally expensive.

The integrators to be introduced later are both second order (though they may be extended to higher order), so in some cases we consider the comparison with the second order explicit Runge-Kutta method, also known as Heun's method. For the general equation $\dot{S}=f(S)$, it takes the form

$$
S^{n+1}=S^{n}+\frac{\Delta t}{2}\left(f\left(S^{n}\right)+f\left(S^{n}+\Delta t f\left(S^{n}\right)\right),\right.
$$

where $\Delta t$ is the time stepsize, $S^{n}$ is an approximation to $S\left(t_{n}\right), t_{n}=t_{0}+n \Delta t$.

All standard schemes conserve linear first integrals such as the total spin. Like the common fourth order explicit Runge-Kutta method, Heun's method conserves none of the other (quadratic) invariant quantities mentioned previously, though conservation of the individual spin lengths can be forced as in [14] if we augment the scheme by scaling each spin vector to length one (i.e. projecting to the unit sphere) at each integration step. Since the violation of the invariant in a single step will be of order no worse than that of the local truncation error, this operation does not change the order of the method. Other integral invariants could also be preserved by a more complicated projection. Such projected Runge-Kutta methods have been studied in [19] based on linearized stability analysis. However, as our experiments will indicate, projection does little to improve the nonlinear stability of the method. 
The implicit midpoint rule, applied to the one-dimensional spin system (2), gives

$$
\begin{aligned}
0 & =\hat{S}_{i}-S_{i}^{n}-\frac{\Delta t}{2} \hat{S}_{i} \times\left(\hat{S}_{i-1}+\hat{S}_{i+1}\right)=: F_{i}(\hat{S}), \quad i=1, \ldots, N \\
S_{i}^{n+1} & =2 \hat{S}_{i}-S_{i}^{n} .
\end{aligned}
$$

The first set of equations is implicit and nonlinear, so we must use an iterative solver such as Newton's method. The Jacobian matrix for Newton iterations applied to (15) is block tridiagonal with elements given by

$$
\begin{aligned}
\frac{\partial F(\hat{S})}{\partial \hat{S}_{i}} & =I-\Delta t \operatorname{skew}\left(\hat{S}_{i-1}+\hat{S}_{i+1}\right), \\
\frac{\partial F(\hat{S})}{\partial \hat{S}_{i \pm 1}} & =\Delta t \text { skew } \hat{S}_{i} .
\end{aligned}
$$

The resulting system can be solved by the block Thomas algorithm. In two dimensions the Jacobian is more complicated, with a block sparse structure analogous to that of the two-dimensional discrete Laplacian.

The implicit midpoint method preserves the symmetry (13) and conserves quadratic first integrals [4] such as the energy and spin lengths, but it is very slow in practice, particularly in two or more space dimensions. This method is also symplectic for canonical Hamiltonian systems, but not for the Landau-Lifshitz equation considered here because of its nonconstant symplectic structure matrix.

3. Geometric Integration Schemes. The two integrators to be introduced in this section are most easily described for the one-dimensional system (2). In both cases the extension to two-dimensions is straightforward, and exceptions for the added coupling in (7) will be noted.

Recall that a splitting $H=H_{1}+H_{2}$ of the Hamiltonian defines a consistent approximation to the flow map via the consecutive solution of the systems

$$
\begin{aligned}
\dot{S} & =J \nabla H_{1} \\
\dot{S} & =J \nabla H_{2}
\end{aligned}
$$

over an interval of length $\Delta t$. If each of the vector fields resulting from the splitting happens to be exactly integrable, then a numerical integration scheme is obtained as the concatenation of the flows of the two systems. For Hamiltonian $H$, let $V_{H}$ represent the corresponding vector field, and let $\exp t V_{H}$ represent the resulting flow map. As pointed out in [25], for a splitting $H=H_{1}+H_{2}$, it is a consequence of the Baker-Campbell-Hausdorff theorem that the local error is of second order:

$$
\exp t V_{H}=\exp t V_{H_{2}} \circ \exp t V_{H_{1}}+O\left(t^{2}\right)
$$

implying that the method is first order accurate. Given such a splitting, and assuming that each of the Hamiltonians $H_{1}$ and $H_{2}$ respects the time-symmetry, then the method defined by

$$
\hat{\Phi}_{t}:=\exp \frac{1}{2} t V_{H_{1}} \circ \exp t V_{H_{2}} \circ \exp \frac{1}{2} t V_{H_{1}}
$$

is a second order, symmetric approximation to the flow map of $H$. Because it is formed from the concatenation of symplectic (or Lie-Poisson, in our case) maps, it will also be a symplectic (Lie-Poisson) map.

A generalization of this approach is obtained by considering arbitrary splittings of the vector field $V_{H}$, i.e. not necessarily derivable as a splitting of the Hamiltonian. If

$$
V_{H}=V_{1}+V_{2},
$$


then the method obtained by the symmetric concatenation of the flows of symmetric vector fields:

$$
\tilde{\Phi}_{t}:=\exp \frac{1}{2} t V_{1} \circ \exp t V_{2} \circ \exp \frac{1}{2} t V_{1}
$$

is again second order and symmetric.

3.1. A Lie-Poisson method. This method will be derived based upon a Hamiltonian splitting. Consider a splitting $H=H_{1}+H_{2}$ of the Hamiltonian $H=-\sum_{i} S_{i} \cdot S_{i+1}$ for the system (2) with

$$
\begin{aligned}
& H_{1}=-\sum_{i \text { odd }} S_{i} \cdot S_{i+1} \\
& H_{2}=-\sum_{i \text { odd }} S_{i} \cdot S_{i-1}
\end{aligned}
$$

Let the integration of (16) propagate the numerical solution from $S^{n}$ to $\hat{S}$. The equations of motion corresponding to $H_{1}$ are

$$
\begin{aligned}
\dot{S}_{i}= & -\operatorname{skew}\left(S_{i}\right) S_{i+1}=S_{i} \times S_{i+1}, \\
\dot{S}_{i+1}= & -\operatorname{skew}\left(S_{i+1}\right) S_{i}=S_{i+1} \times S_{i}, \\
& i=1, \cdots, N \text { and } i \text { odd. }
\end{aligned}
$$

These equations can be easily solved in pair. In fact, for odd index $i$ we have

$$
\begin{aligned}
S_{i}+S_{i+1} & \equiv \text { const }=S_{i}^{n}+S_{i+1}^{n}=B_{i} \\
\dot{S}_{i} & =S_{i} \times S_{i+1}=S_{i} \times\left(B_{i}-S_{i}\right)=\left(\text { skew } B_{i}\right) S_{i}, \\
\dot{S}_{i+1} & =S_{i+1} \times S_{i}=S_{i+1} \times\left(B_{i}-S_{i+1}\right)=\left(\operatorname{skew} B_{i}\right) S_{i+1} .
\end{aligned}
$$

We can then solve the resulting differential equations exactly for a timestep $\Delta t$ :

$$
\begin{aligned}
\hat{S}_{i} & =e^{\Delta t \text { skew } B_{i}} S_{i}^{n} \\
\hat{S}_{i+1} & =e^{\Delta t \text { skew } B_{i}} S_{i+1}^{n} .
\end{aligned}
$$

Thus the subsystem corresponding to Hamiltonian $H_{1}$ is exactly integrable. Similarly, the subsystem on $H_{2}$ is exactly integrable. We use these two flows successively to develop an approximate integrator. The complete timestepping procedure can be summarized as follows: we first sweep through the odd indices, sum each spin vector with its neighbor to the right to get $B_{i}$, and compute the rotation matrix $\exp \left(\Delta t \operatorname{skew} B_{i}\right)$, applying this rotation to both of the spin vectors. We then repeat the sweep, this time coupling each of the odd spins with its neighbor to the left. Note that this procedure involves many independent tasks which could be effectively computed in parallel.

To extend this method to the two-dimensional anisotropic system (4) with $h=1$, we utilize Red-Black ordering of the domain, defining the sets

$$
\text { reds }=\{(i, j) \mid i+j \text { even }\}, \quad \text { blacks }=\{(i, j) \mid i+j \text { odd }\} .
$$

For integrating the discretization (4), we make use of the symmetric splitting

$$
H=\frac{1}{2} H_{1}+\frac{1}{2} H_{2}+\frac{1}{2} H_{3}+\frac{1}{2} H_{4}+H_{5}+\frac{1}{2} H_{4}+\frac{1}{2} H_{3}+\frac{1}{2} H_{2}+\frac{1}{2} H_{1},
$$


where

$$
\begin{aligned}
H_{1} & =\sum_{\text {reds }} S_{i, j} \cdot S_{i+1, j}, & H_{2} & =\sum_{\text {reds }} S_{i, j} \cdot S_{i-1, j}, \quad H_{3}=\sum_{\text {reds }} S_{i, j} \cdot S_{i, j-1}, \\
H_{4} & =\sum_{\text {reds }} S_{i, j} \cdot S_{i, j+1}, & H_{5} & =\sum_{i, j} S_{i, j} \cdot D S_{i, j} .
\end{aligned}
$$

The Hamiltonian $H_{5}$, which is the Hamiltonian of the Euler equation, is not integrable; we follow the approach used by Reich [15] and further split this term as follows: $H_{5}=\frac{1}{2} H_{5 a}+\frac{1}{2} H_{5 b}+H_{5 c}+\frac{1}{2} H_{5 b}+\frac{1}{2} H_{5 a}$, where, denoting the three components of $S_{i j}$ by $S_{i j}^{1}, S_{i j}^{2}$, and $S_{i j}^{3}$, we have

$$
H_{5 a}=\sum_{i, j} d_{1}\left(S_{i j}^{1}\right)^{2}, \quad H_{5 b}=\sum_{i, j} d_{2}\left(S_{i j}^{2}\right)^{2}, \quad H_{5 c}=\sum_{i, j} d_{3}\left(S_{i j}^{3}\right)^{2} .
$$

Each of these terms yields an integrable system involving only rotations. (Note that for easy plane anisotropy (8), the terms $H_{5 a}$ and $H_{5 b}$ vanish.) Specifically, this method requires 13 rotations in general per spin vector per time step, with the result that it is slow when implemented sequentially. However, the scheme involves many tasks that can be executed in parallel. Despite the large number of parts of this splitting, the scheme appears to retain a reasonable degree of numerical stability.

For the Roberts discretization (7) in the anisotropic case, the appropriate Hamiltonian splitting is simplified, and would rely on the exact integration of pairs of neighboring spins of the form

$$
\begin{aligned}
\frac{d}{d t} S_{i j} & =S_{i j} \times M S_{k l}, \\
\frac{d}{d t} S_{k l} & =S_{k l} \times M S_{i j} .
\end{aligned}
$$

Since $M$ is symmetric, we can write

$$
\begin{aligned}
\frac{d}{d t} S_{k l} & =S_{k l} \times M S_{i j} \\
& =-M S_{i j} \times S_{k l} \\
& =-S_{i j} \times M S_{k l} \\
& =-\frac{d}{d t} S_{i j} .
\end{aligned}
$$

Thus we can write $S_{i j}+S_{k l}=\Delta=$ const, and this relation can be used to reduce the first differential equation to

$$
\frac{d}{d t} S_{i j}=S_{i j} \times M\left(\Delta-S_{i j}\right)
$$

which is essentially the Euler equation. In other words, we do not have a means of exactly integrating the splitting, and so must rely on a further decomposition of the problem. It is therefore likely that the previous approach, based on solving (4), is more appropriate.

3.2. A staggered Red-Black method. An alternative method also uses Red-Black ordering of the domain and additionally staggered computation of red and black grid points. This approach leads to a method with similar numerical conservation properties to the reversible energy-momentum method (see [7]) proposed for integrating canonical Hamiltonians, but the method of this section is explicit, requiring only the solution of as many $3 \times 3$ linear algebraic systems as the number of spin sites.

Consider again the one-dimensional semidiscrete system with an even number of spin sites. Define

$$
P_{i} \equiv S_{2 i-1}, \quad Q_{i} \equiv S_{2 i}, \quad i=1, \ldots, N / 2,
$$


so that we have the equations:

$$
\frac{d}{d t} P_{i}=P_{i} \times\left(Q_{i-1}+Q_{i}\right), \quad \frac{d}{d t} Q_{i}=Q_{i} \times\left(P_{i}+P_{i+1}\right), \quad i=1, \ldots, N / 2 .
$$

Define a vector field splitting by

$$
V_{1}=\left[\begin{array}{c}
P_{1} \times\left(Q_{N / 2}+Q_{1}\right) \\
0 \\
P_{2} \times\left(Q_{1}+Q_{2}\right) \\
0 \\
P_{3} \times\left(Q_{2}+Q_{3}\right) \\
\vdots \\
P_{\frac{1}{2} N} \times\left(Q_{\frac{1}{2} N-1}+Q_{\frac{1}{2} N}\right) \\
0
\end{array}\right], \quad \text { and } \quad V_{2}=\left[\begin{array}{c}
0 \\
Q_{1} \times\left(P_{1}+P_{2}\right) \\
0 \\
Q_{2} \times\left(P_{2}+P_{3}\right) \\
0 \\
Q_{3} \times\left(P_{3}+P_{4}\right) \\
\vdots \\
Q_{\frac{1}{2} N} \times\left(P_{\frac{1}{2} N}+P_{1}\right)
\end{array}\right]
$$

It is easy to see that each of these vector fields is exactly integrable; all that is required is the computation of a rotation (as a matrix exponential) for each spin vector at each timestep. We implement the symmetric approximation method $\tilde{\Phi}_{t}$ using the pair of vector fields $V_{1}$ and $V_{2}$.

The resulting method will not be symplectic, however, since the vector field splitting does not correspond to a splitting of the Hamiltonian. Since the method is only symmetric, it seems reasonable to replace the rotations with second order Padé(1,1) approximations to the corresponding matrix exponential (preserving the symmetry). Recall that the Padé(1,1) approximation of $e^{h A}$ is just the Cayley transform:

$$
e^{h A} \approx\left(I-\frac{1}{2} h A\right)^{-1}\left(I+\frac{1}{2} h A\right)
$$

This proves to reduce the cost of the step update considerably, and may be particularly important in two or three space dimensions.

By simplifying the resulting equations-eliminating the $P$ approximations at half steps in time and those of $Q$ at whole steps - the described method can be seen to be equivalent to the following staggered timestepping scheme:

$$
\begin{gathered}
Q_{i}^{n+\frac{1}{2}}=Q_{i}^{n-\frac{1}{2}}+\frac{\Delta t}{2}\left(Q_{i}^{n+\frac{1}{2}}+Q_{i}^{n-\frac{1}{2}}\right) \times\left(P_{i}^{n}+P_{i+1}^{n}\right), \\
P_{i}^{n+1}=P_{i}^{n}+\frac{\Delta t}{2}\left(P_{i}^{n+1}+P_{i}^{n}\right) \times\left(Q_{i-1}^{n+\frac{1}{2}}+Q_{i}^{n+\frac{1}{2}}\right) .
\end{gathered}
$$

In general, we need to solve a linear system at each step, computing an explicit, orthogonal update such as

$$
Q_{i}^{n+\frac{1}{2}}=\left(I-\frac{\Delta t}{2} \operatorname{skew} B\right)^{-1}\left(I+\frac{\Delta t}{2} \operatorname{skew} B\right) Q_{i}^{n-\frac{1}{2}},
$$

where $B=P_{i}^{n}+P_{i+1}^{n}$. The update for $P$ is similar.

For the purpose of starting, or to have a mapping of phase space, we introduce an approximation to $Q$ at timesteps and decompose (23) into

$$
\begin{aligned}
Q_{i}^{n+\frac{1}{2}} & =Q_{i}^{n}+\frac{\Delta t}{2} Q_{i}^{n+\frac{1}{2}} \times\left(P_{i}^{n}+P_{i+1}^{n}\right), \\
Q_{i}^{n+1} & =Q_{i}^{n+\frac{1}{2}}+\frac{\Delta t}{2} Q_{i}^{n+\frac{1}{2}} \times\left(P_{i}^{n+1}+P_{i+1}^{n+1}\right) .
\end{aligned}
$$

In this form we recognize the scheme as the second order Lobatto IIIA-B partitioned Runge-Kutta method [19] applied with the indicated partitioning of the variables. 
Extension of this scheme to the 2D isotropic case is straightforward. After Red-Black splitting, we again obtain two vectors $P$ and $Q$ of spins. For any spin vector $P_{i, j}$, we will have to compute an update such as (25), but with $B$ consisting of the sum of all neighboring spin sites.

When extending the staggered Red-Black scheme to treat an anisotropy such as (8), the Roberts discretization presents a distinct advantage over the lattice Landau-Lifshitz equation. For the standard lattice Landau-Lifshitz equation (4), we need to handle the rigid body term $S_{i} \times D S_{i}$ which arises in the right hand side. For a symmetric integrator, we can utilize the Padé(1,1) approximation for this term, leading to the staggered scheme (in the 1D case)

$$
\begin{aligned}
Q_{i}^{n+\frac{1}{2}}=Q_{i}^{n-\frac{1}{2}}+\frac{\Delta t}{2}\left(Q_{i}^{n+\frac{1}{2}}+Q_{i}^{n-\frac{1}{2}}\right) \times\left(P_{i}^{n}+P_{i+1}^{n}\right) \\
+ \\
+\frac{\Delta t}{2}\left(Q_{i}^{n+\frac{1}{2}}+Q_{i}^{n-\frac{1}{2}}\right) \times D\left(Q_{i}^{n+\frac{1}{2}}+Q_{i}^{n-\frac{1}{2}}\right), \\
P_{i}^{n+1}=P_{i}^{n}+\frac{\Delta t}{2}\left(P_{i}^{n+1}-P_{i}^{n}\right) \times\left(Q_{i-1}^{n+\frac{1}{2}}+Q_{i}^{n+\frac{1}{2}}\right) \\
+\frac{\Delta t}{2}\left(P_{i}^{n}+P_{i}^{n+1}\right) \times D\left(P_{i}^{n}+P_{i}^{n+1}\right) .
\end{aligned}
$$

This requires the solution of a three-dimensional nonlinear system at each spin site, at each step.

Using the Roberts discretization in the anisotropic case, on the other hand, merely modifies the form of the linear systems that must be solved: no implicit solve is required.

3.3. Conserved quantities of the Lie-Poisson and staggered Red-Black schemes. Both the LiePoisson and staggered Red-Black schemes conserve the lengths of spin vectors. Both schemes also conserve the total spin (for the isotropic problem with periodic boundary conditions), and-somewhat surprisingly, given its simplicity and its semi-explicit form - the staggered scheme conserves the energy (for both isotropic and anisotropic cases and with either type of boundary conditions).

Recall that a Hamiltonian system $H$ possesses an integral $R$ provided the Poisson bracket of $R$ with $H$ vanishes:

$$
\{R, H\}=0 .
$$

For the one-dimensional spin-chain, let $T=\sum_{i} S_{i}$. We have, for each of the three components $T_{(l)}, l=1,2,3$ of $R$,

$$
\begin{aligned}
\left\{T_{(l)}, H\right\} & =\left(\sum_{i=1}^{N} S_{i} \times\left(S_{i-1}+S_{i+1}\right)\right)_{(l)} \\
& =\left(\sum_{i} S_{i} \times S_{i-1}-\sum_{i} S_{i+1} \times S_{i}\right)_{(l)} \\
& =0,
\end{aligned}
$$

by use of the periodic boundary conditions. Similarly, for the Lie-Poisson scheme,

$$
\left\{T_{(l)}, H_{1}\right\}=\left(\sum_{k=1}^{N / 2}\left(S_{2 k-1} \times S_{2 k}+S_{2 k} \times S_{2 k-1}\right)\right)_{(l)}=0,
$$

and, likewise, $\left\{T_{(l)}, H_{2}\right\}=0$. The total spin is thus a conserved quantity of each step of the scheme. This argument is easily generalized to the isotropic problem on a 2D lattice. (The total spin is not an integral of the anisotropic system.) 
Next, we turn to the staggered discretization. Recall that $R$ is an integral of a vector field $V$ provided $\nabla R^{T} V \equiv 0$. For example, for the energy $H$, we automatically have

$$
\nabla H^{T} J \nabla H=0
$$

since $J$ is skew-symmetric. Considering now the one-dimensional isotropic case, observe that

$$
\nabla H^{T} V_{1}=\sum_{i=1}^{\frac{1}{2} N} P_{i} \cdot\left(P_{i} \times\left(Q_{i}+Q_{i+1}\right)\right)=0,
$$

with a similar relation for $V_{2}$, so the Hamiltonian is conserved under the vector field splitting. Conservation of the total spin, and - in the isotropic case - of the energy, can be viewed as a consequence of more general integral-preservation properties of the Lobatto IIIA-B PRK scheme. Both of these integrals can be written in the form $P^{T} A Q$, for $A$ a constant matrix. A theorem of Reich [15] states that such integral invariants are conserved by partitioned Runge-Kutta methods whose coefficients satisfy a certain algebraic condition which is equivalent to the condition for such a method to be symplectic when applied to a canonical Hamiltonian system-and which is satisfied by the Lobatto IIIA-B PRK pair.

In this context, we observe another superior feature of the Roberts discretization as compared to the Lattice Landau-Lifshitz formulation. For the former discretization, the energy retains the form of a bilinear invariant in $P$ and $Q$, even in the anisotropic case, and thus remains a conserved quantity under discretization. The discretization (4), in addition to requiring more computational work, does not lead to an energy conserving method when treated with Red-Black splitting.

4. Numerical experiments and discussion. In this section we present numerical experiments used to validate the integrators described in the previous sections: the explicit Runge-Kutta methods (the fourth order method (RK4) and Heun's method (H) given by (14)), the projected explicit Runge-Kutta methods (the projected fourth order (RK4p) and projected Heun's (Hp) methods), the implicit midpoint (IM) given by (15), the Lie-Poisson method (LP) of $\S 3.1$, and the staggered Red-Black scheme (RB) of $\S 3.2$. Two examples are considered: the first one is the one-dimensional isotropic system with the exact solution given by (3) and the other is the problem of anisotropic vortices on a two-dimensional lattice.

4.1. Isotropic spin chain on a one-dimensional lattice. For comparing the accuracy and stability of the integrators it is handy to integrate the one-dimensional Heisenberg chain (2) with the exact solution. We choose the parameters in equation (3) as $\phi=\pi / 3, p=2 \pi / N, a=[1,2,-1]^{T} / \sqrt{6}, b=[2,1,4]^{T} / \sqrt{21}$, and $c=a \times b$, where $N$ is the number of spins on the chain.

We first consider a chain of $N=20$ spins, integrating with a time stepsize of $\Delta t=0.02$ over a short time period (2000 time units). Figure 1 illustrates the error evolution of the various methods. Here the error is defined as the Euclidean norm of the difference between the computed and exact spin vectors at an arbitrarily selected site $(i=14)$ in the spin chain. It can be seen that all of the methods have similar error growths. The IM and Hp methods produce more accurate results than the other methods for the short time period. However, we shall see that in the long term, both the $\mathrm{H}$ and Hp methods blow up, while the IM method is very expensive from the computational point of view. In fact, in order to generate the results shown in the figure the IM method took more than 20 times as long as that the RB scheme did, making it feasible to use the RB method with an order of magnitude smaller stepsize in place of the IM method. The operations counts for all of the schemes are summarized in Table 1. (In the table, $N$ is the number of spins in the system, and $m$ is the number of Newton iterations required.)

Now we look at the conservative properties of these schemes. The error in the $z$-component of the total spin is shown in Figure 2. Apparently the total spin is conserved to rounding error accuracy by all methods. 
TABLE 1

Operations counts

\begin{tabular}{|l|r|r|r|r|}
\hline Integrator & $1-D$ & $2-D$ & $3-D$ & Aniso. \\
\hline Heun & $39 N$ & $51 N$ & $63 N$ & $+2 N$ \\
Implicit Midpoint & $(19 m+407) N$ & & & \\
Red-Black & $86 N$ & $92 N$ & $98 N$ & $+N$ \\
Lie-Poisson & $94 N$ & $221 N$ & $348 N$ & $+41 N$ \\
\hline
\end{tabular}

More interesting is the conservation of individual spin length at an arbitrarily selected site $(i=14)$ shown in Figure 3. The individual spin length is conserved by all of the methods but the (unprojected) Heun method. Figure 4 shows the energy conservation properties for the schemes. Using the IM and RB methods, the Hamiltonian is conserved to the level of roundoff error. The deviation of the energy obtained with the Lie-Poisson method oscillates but appears to stay bounded in the short term. (The oscillation is reduced somewhat in frequency in the figure by sampling every five steps in order to show the error of the Hp method in the same figure.) To show the stability of the schemes, we next integrate the system of the same number of spins with $\Delta t=0.02$ for a much longer term. Figure 5 shows the results obtained with the H, Hp, LP, and $\mathrm{RB}$ methods. It can readily seen that both the $\mathrm{H}$ and $\mathrm{Hp}$ methods blow up at around $t=10^{5}$ while the LP and RB schemes retain good stability and accuracy.

4.2. Anisotropic vortices on a two-dimensional lattice. The study of vortices and antivortices in Heisenberg ferromagnets has attracted considerable interest from physicists in recent years, e.g. see [12, 14, 24, 23]. Among many applications, these problems are representative of the dependence of macroscopic coherent structures on microscopic interactions [21]. The motion of vortex pairs in spin lattices with easyplane anisotropy (8) is developed in [21]. Mertens et al. [12] present theoretical and computational results for various combinations of vortices and anti-vortices, including some very interesting vortex core trajectories. In [24] Wysin et al. showed that such vortices in easy plane magnets have an effective mass and momentum. Wysin [23] also determined theoretically the threshold on various grid structures for the parameter $\delta$ in (8) between stable in-plane and out-of-plane vortices. In [14] the interaction of vortex pairs and vortexantivortex pairs governed by a modified Landau-Lifshitz equation were studied numerically. Elementary forms for planar vortices are developed in [14]. An antivortex is the complex conjugate of a planar vortex field. Additionally, planar vortices (antivortices) have a positive or negative orientation (for vortices, whether they are counterclockwise or clockwise, respectively). The vortices admitted by easy-plane anisotropy have as well a positive- or negatively oriented out-of-plane component near the vortex core, with a spin vector at the core having no component in the plane. There are thus six types of vortical motion (considering antivortices as a type of vortical motion) in easy-plane magnets.

We consider two vortex systems in this section: a vortex-vortex pair with alike out-of-plane orientation and opposite rotational orientation, as well as a vortex-antivortex pair with opposite out-of-plane and rotational orientations. For the numerical experiments of this section, we used the homogeneous Dirichlet boundary conditions and easy plane anisotropy (8) with $\delta=0.1$ for the vortex-antivortex pair and $\delta=0.2$ for the vortex-vortex pair. These values of the anisotropy parameter admit stable out-of-plane vortices [23]. Due to the significantly increased workload for the IM method in two dimensions, we felt that the method would not be competitive and did not implement it. We instead compare results with the classical fourth-order Runge-Kutta method and a projected version of the same.

To obtain initial vortex (antivortex) solutions, we started with elementary planar rotational fields with no out-of-plane component (these were obtained by projecting vortex solutions of the Skyrmian system of [14] into the plane). Then, following [24], a small out-of-plane component was given to the four spin sites 
surrounding the vortex (antivortex) core. Next the equations of motion were integrated with an additional Gilbert damping term using the RK4 scheme. The reader is referred to [3] for the details and properties of Gilbert damping. For our vortex-antivortex simulation, a value $\gamma=0.3$ of the damping parameter was used and the integration was performed for 500 steps of size $\Delta t=0.1$. For the the vortex-vortex simulation we damped with $\gamma=0.5$ for 30000 steps of size $\Delta t=0.001$. We used the following heuristic method of determining the core location during the evolution of the system. Since the vortex and antivortex had opposite out-of-plane components, we searched for the maximum and minimum out-of-plane components, then determined the adjacent cell with maximum sum of out-of-plane component magnitudes. Finally the location of the core was taken as a mass average over the 9 adjacent cells (16 spins), with discrete mass given (through trial-and-error) by

$$
\Delta m_{i j}=\left|S_{i j}^{z}\right|^{5},
$$

where $S_{i j}^{z}$ is the $z$-component of the spin at grid location $(i, j)$.

The experiments were conducted on various sizes of grids. The following results for the vortex-antivortex pair were obtained on a $50 \times 50$ lattice. The vortex and antivortex were initialized in the center of two grid cells located on the axis, 14 grid spacings apart. Figures 6, 7 and 8 illustrate approximately one period of vortex-antivortex motion computed with the RK4p, RB, and LP integrators, respectively. The results are nearly identical to appearance, and exhibit a hexagonal trajectory similar to that found in [12]. Figure 9 shows the evolution of the vortex-antivortex pair during approximately one half period of motion. In the figure, the surface plot represents the $z$-components of spins and the vector field shows the $x$ - and $y$ components. For this case, we found that the system is very stable and all of the projected Runge-Kutta, the Lie-Poisson, and the staggered Red-Black methods produce fairly stable and accurate results even for a very long time interval.

The situation is quite different for the vortex-vortex system. For comparison of stability, a vortex-vortex pair with alike out-of-plane component and opposite rotational direction were integrated on a $64 \times 64$ grid. Figure 10 illustrates the conservation of Hamiltonian for the various methods. In the short term (about 0.46 period of revolution of the vortices), with a stepsize of $\Delta t=0.025$, the bottom pane of the figure shows that the Hp method blows up at time 2500. We also see that the integrations using RB and LP schemes were stable over the integration interval. On the long time interval, the top pane shows over about 5.5 periods of the revolution of the vortices that for stepsizes of $0.1,0.05$ and 0.025 , the RK4p scheme eventually blows up, whereas the Hamiltonian computed by the RB scheme remains on the order of roundoff error for all three stepsizes. (Note that we have not run the LP integrator for this long time interval because it is too expensive.) As a final illustration of the long term behavior of the RB scheme, Figure 11 shows the core trajectory of a vortex in a vortex-antivortex pair, through approximately 10 periods of revolution of the system.

To conclude this section, we list in Table $2 \mathrm{CPU}$ times of some vortex-vortex simulations using our methods. In the table, $K$ is the number of time steps taken. In general we see that the RB scheme requires about one-fourth the time of the fourth-order RK scheme, and that the LP method requires about eight times as long as the RB method. It is important to point out that these times are dependent on the particular implementation that we have used, and that a great deal of efficiency improvements would be possible in the computation of the matrix exponentials of the LP method.

5. Conclusions. Two new integrators have been developed in the previous sections for simulating classical Heisenberg spin systems. One of the new integrators, the Lie-Poison method, was derived based upon a Hamiltonian splitting; it conserves all of the integrals (spin-length and total spin) of the differential 
TABLE 2

CPU Time Comparison

\begin{tabular}{|l|c|l|r|r|}
\hline Integrator & Lattice & $\Delta t$ & $K$ & time, hrs \\
\hline RK4p & $64 \times 64$ & 0.1 & 300000 & 11.9 \\
RK4p & $64 \times 64$ & 0.05 & 600000 & 24.7 \\
RK4p & $64 \times 64$ & 0.025 & 1200000 & 48.3 \\
RK4p & $256 \times 256$ & 0.05 & 1200000 & $>485.0$ \\
\hline RB & $64 \times 64$ & 0.1 & 300000 & 2.5 \\
RB & $64 \times 64$ & 0.05 & 600000 & 5.0 \\
RB & $64 \times 64$ & 0.025 & 1200000 & 10.1 \\
RB & $256 \times 256$ & 0.05 & 1200000 & 168.0 \\
\hline LP & $64 \times 64$ & 0.05 & 100000 & 6.6 \\
LP & $64 \times 64$ & 0.025 & 100000 & 6.6 \\
\hline
\end{tabular}

equations but the energy. The other integrator was derived based upon a Red-Black splitting and staggered timestepping. This method is time symmetric and conserves all the available first integrals.

The two new integrators were applied to both 1D and 2D spin systems and compared with explicit RungeKutta methods, their projected variants, and the implicit midpoint scheme. The results show that both the Runge-Kutta methods and their projected variants exhibit numerical instability after long times while the implicit midpoint is computationally too expensive to be practical. On the other hand, the numerical results have demonstrated the efficiency and long time stability of both the Lie-Poisson and staggered Red-Black schemes. We observed no qualitative difference between the time-reversible and Lie-Poisson schemes. For the 2D system, the staggered Red-Black scheme scheme requires about one-fourth the time of the projected fourth-order Runge-Kutta scheme. If higher order methods are needed, the technique of [25] could be used to construct reasonable fourth order or sixth order time-reversible integrators.

Acknowledgment. The authors are grateful to Gary Wysin at the Kansas State University for his useful comments and information on spin systems.

\section{REFERENCES}

[1] J. Wisdom and M. Holman, Symplectic maps for the N-body problem, Astron. J., 102:1528-1538, 1991.

[2] J. E. Marsden, Lectures on Mechanics, Cambridge University Press, 1992.

[3] A. Bloch and P.S. Krishnaprasad and J.E. Marsden and T. Ratiu, The Euler-Poincaré Equations and Double Bracket Dissipation, preprint, 1996.

[4] G. Cooper, Stability of Runge-Kutta methods for trajectory problems, IMA J. Num. Anal., 7:1-13, 1987.

[5] D. J. Dichmann and J. H. Maddocks, An Impetus-Striction Simulation of the Dynamics of an Elastica, J. Nonlinear Science, 6:271-292, 1996.

[6] L.D. Faddeev and L.A. Takhtajan, Hamiltonian Methods in the Theory of Solitons, Springer-Verlag, Berlin, 1987.

[7] O. Gonzales and J. Simo, On the Stability of Symplectic and Energy-Momentum Algorithms for Nonlinear Hamiltonian Systems with Symmetry, Comp. Meth. Appl. Mech. Engr., 1996 (to appear).

[8] E. Hairer, Backward error analysis of numerical integrators and symplectic methods, Annals of Numerical Mathematics, $1: 107-132,1994$.

[9] B. Leimkuhler and G. Patrick, A Symplectic Integrator for Riemannian Manifolds, J. Nonlinear Science, 1996 (to appear).

[10] B. Leimkuhler and S. Reich, Symplectic Integration of Constrained Hamiltonian Systems, Mathematics of Computation, 63:589-605, 1994

[11] R. McLachlan, Numerical Solution of Hamiltonian Wave Equations, Numerische Mathematik, 66:465-492, 1994.

[12] F.G. Mertens, G.M. Wysin, A.R. Völkel, A.R. Bishop, and H.J. Schnitzer, Cyclotron-like Oscillations and Boundary Effects in the 2-Vortex Dynamics of Easy-Plane Magnets, in Bayreuth Workshop on Nonlinear Coherent Structures, 1993.

[13] P.J. Olver, Applications of Lie Groups to Differential Equations, Springer-Verlag, New York, 1986.

[14] N. Papanicolaou and W.J. Zakrewski, Dynamics of Interacting Magnetic Vortices in a Model Landau-Lifshitz Equation, Physica D, Nonlinear Phenomena, 80:225-245, 1995.

[15] S. Reich, Momentum conserving symplectic integrators, Physica D, 76:375-383, 1994.

[16] J.A.G. Roberts and C.J. Thompson, Dynamics of the Classical Heisenberg Spin Chain, Technique Report 11-1987, 1987, 
Mathematics Department, University of Melbourne, Parkville, Victoria 3052, Australia.

[17] J. Frutos, T. Ortega and J.M Sanz-Serna, A Hamiltonian explicit algorithm with spectral accuracy for the 'good' Boussinesq equation, Comp. Meth. Appl. Mech. Engrg., 80:417-423, 1990.

[18] J.M. Sanz-Serna and M.P. Calvo, Numerical Hamiltonian Problems, Vol. 7 Applied Mathematics and Mathematical Computation, Chapman and Hall, London, 1994.

[19] G. Sun, Symplectic Partitioned Runge-Kutta Methods, J. Comput. Math., 11:365-372, 1993.

[20] L. A. Takhtajan, Integration of the continuous Heisenberg spin chain through the inverse scattering method, Phys. Lett. A, 64:235-237, 1977.

[21] A.R. Völkel, F.G. Mertens, A.R. Bishop, and G.M. Wysin, Motion of Vortex Pairs in the Ferromagnetic and Antiferromagnetic Anisotropic Heisenberg Model, Physical Review B, 43:5992-6005, 1991.

[22] A.R. Völkel, F.G. Mertens, G.M. Wysin, A.R. Bishop, and H.J. Schnitzer, Collective Variable Approach for a Magnetic N-Vortex System, in Bayreuth Workshop on Nonlinear Coherent Structures, 1993.

[23] G.M. Wysin, Instability of In-Plane Vortices in Two-Dimensional Easy-Plane Ferromagnets, Physical Review B, 49:8780$8789,1994$.

[24] G.M. Wysin, F.G. Mertens, A.R. Völkel, and A.R. Bishop, Mass and Momentum for Vortices in Two-Dimensional EasyPlane Magnets, in Bayreuth Workshop on Nonlinear Coherent Structures, 1993.

[25] H. Yoshida, Construction of Higher Order Symplectic Integrators, Phys. Lett. A, 150:262-280, 1990. 


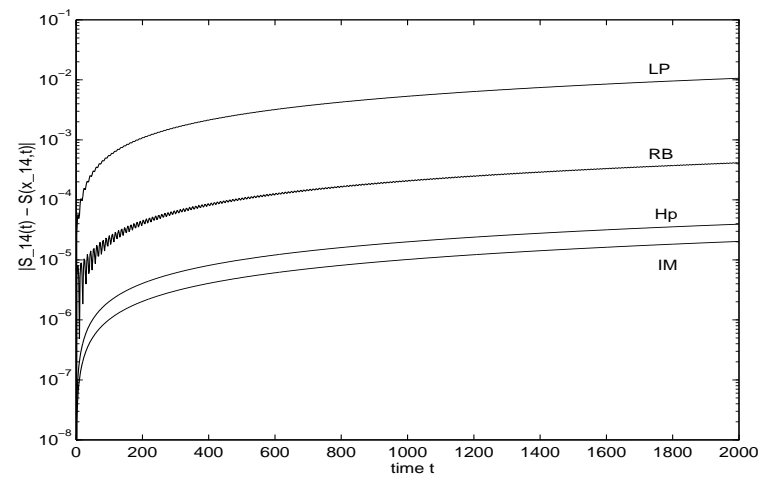

FIG. 1. A comparison of the error for the one-dimensional isotropic spin chain.
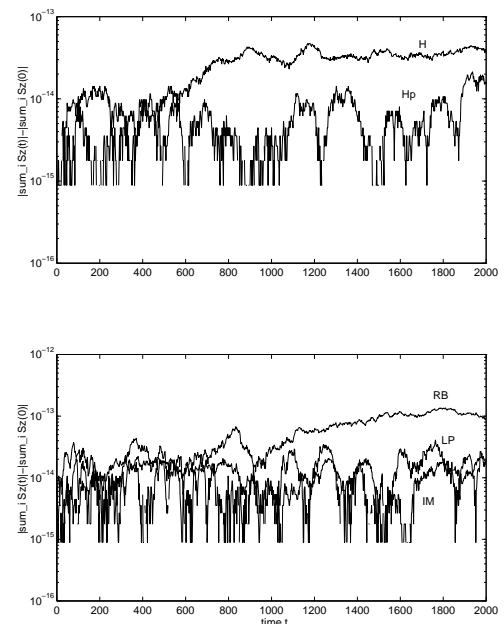

FIG. 2. Total spin conservation properties (z-component) for the various schemes.
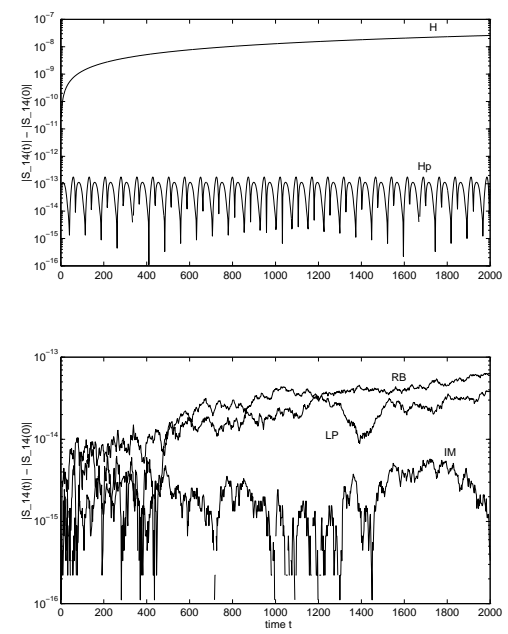

FIG. 3. Individual length conservation properties of the various schemes. 


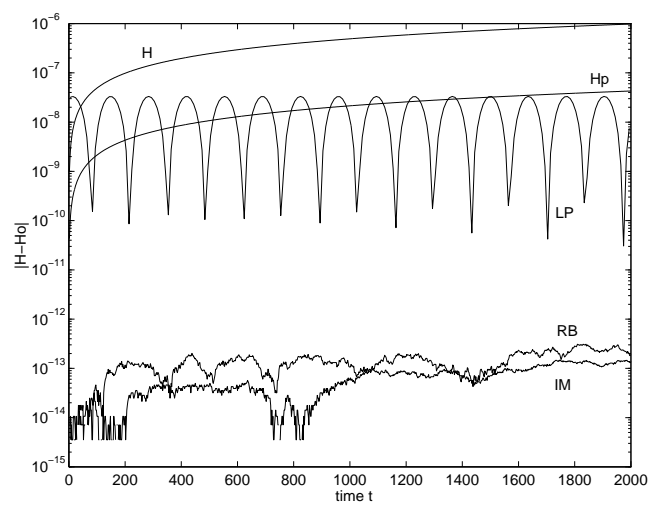

FIG. 4. Energy conservation properties for the various schemes.

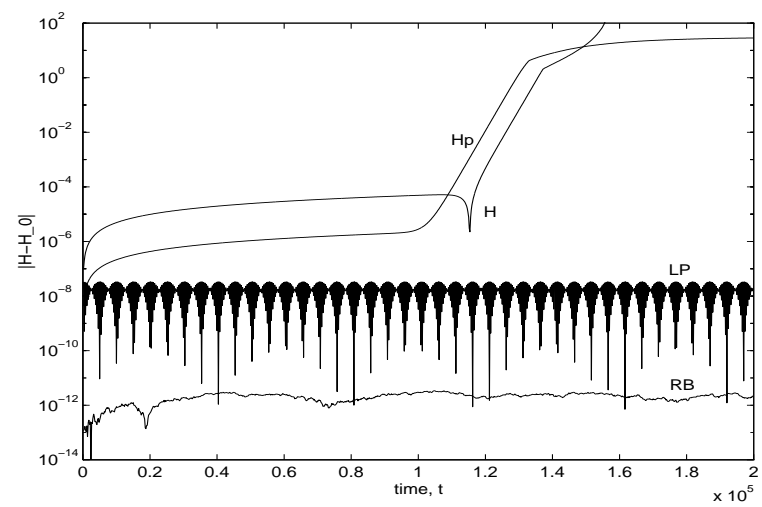

FIG. 5. Energy conservation properties of the various schemes for the long term.

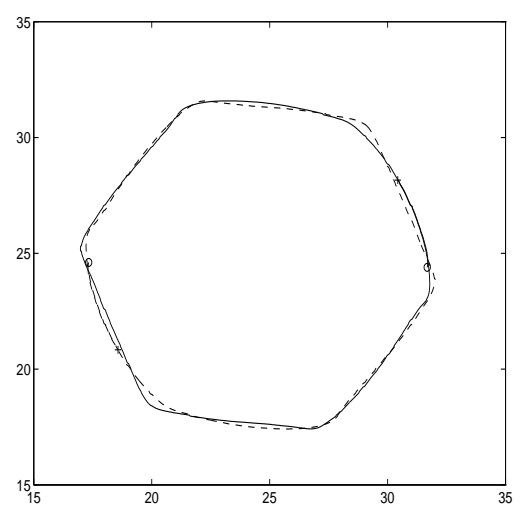

FIG. 6. Vortex-antivortex core trajectories computed by the projected fourth order Runge-Kutta method. The initial and final core locations are indicated with 'o' and ' + ', respectively. The solid line is for the vortex and the dashed line for the antivortex. 


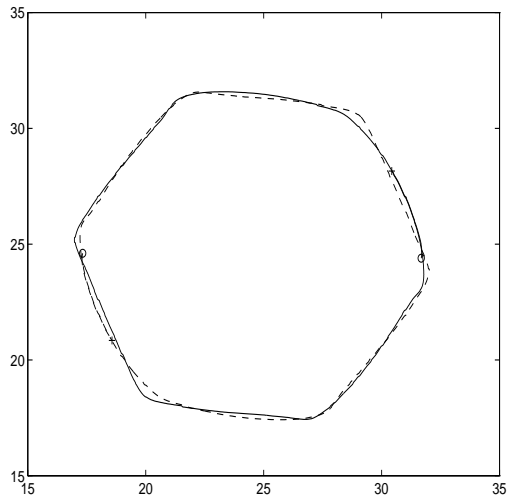

FIG. 7. Vortex-antivortex core trajectories computed by the staggered Red-Black scheme. The initial and final core locations are indicated with ' $O$ ' and ' + ', respectively. The solid line is for the vortex and the dashed line for the antivortex.

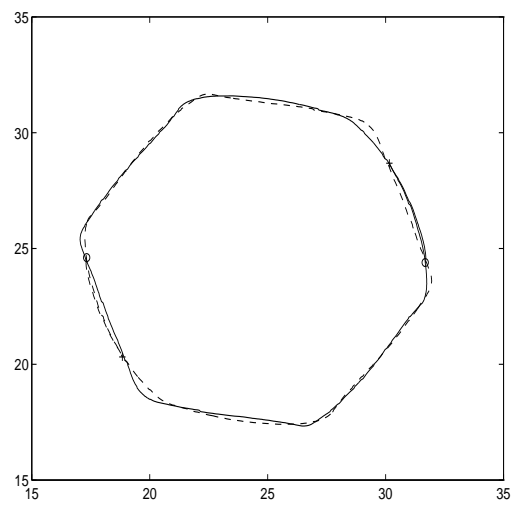

FIG. 8. Vortex-antivortex core trajectories computed by the Lie-Poisson method. The initial and final core locations are indicated with 'o' and ' + ', respectively. The solid line is for the vortex and the dashed line for the antivortex. 



FIG. 9. The evolution of the vortex-antivortex pair over about one half period of motion (from top to bottom and left to right). The surface plot represents the $z$ components of spins and the vector field shows the $x$ and $y$ components. 

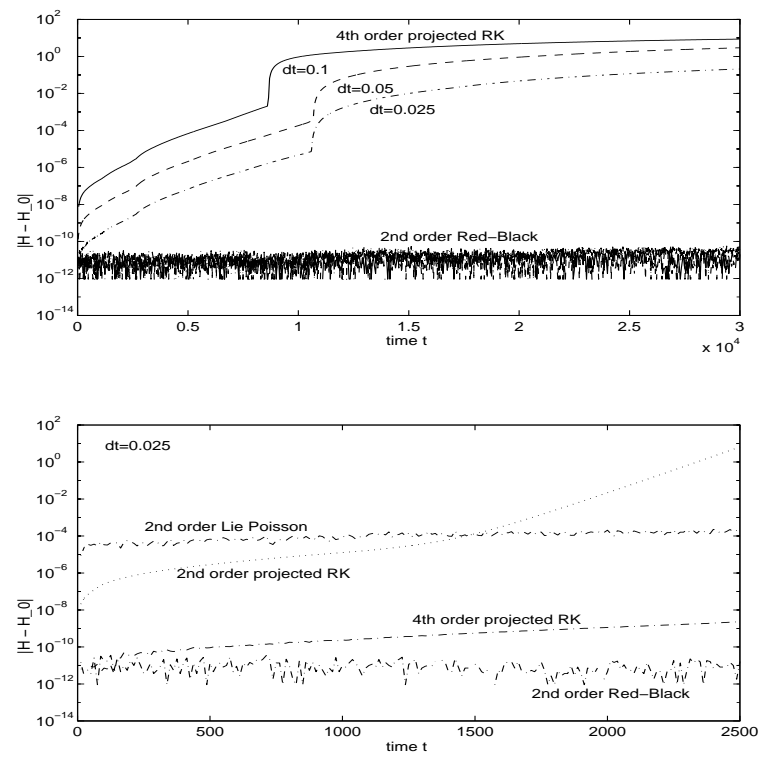

FIG. 10. Hamiltonian conservation. a) projected $R K 4$ vs $R B$ for stepsizes of $\Delta t=0.1,0.05,0.025$ b) Lie-Poisson integrator and projected $R K 2$ vs $R K 4$ and $R B$ for stepsize of $\Delta t=0.025$.

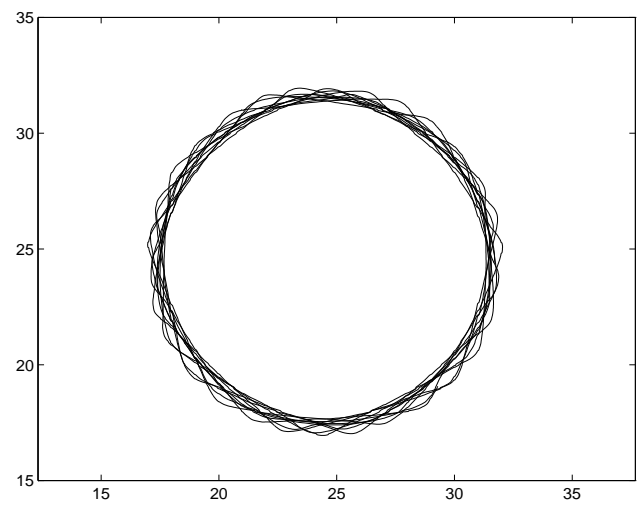

FIG. 11. Revolution over about 10 periods of the vortex-antivortex pair obtained with the staggered Red-Black scheme. 